\title{
La importancia de Palenque, Chiapas, para la conservación de los murciélagos de México
}

\section{The importance of Palenque, Chiapas, for the conservation of Mexican bats}

\author{
Luis Gerardo Avila-Torresagatón ${ }^{1}$, Mircea Hidalgo-Mihart ${ }^{1 \bowtie}$ y José Antonio Guerrero² \\ ${ }^{1}$ División Académica de Ciencias Biológicas, Universidad Juárez Autónoma de Tabasco. Carretera Villahermosa-Cárdenas Km. 0.5 s/n, entronque \\ Bosques de Saloya 86150 Villahermosa, Tabasco, México. \\ ${ }^{2}$ Facultad de Ciencias Biológicas, Universidad Autónoma del Estado de Morelos. Avenida Universidad 1001, Col. Chamilpa 62210 Cuernavaca, \\ Morelos, México. \\ \midalgo@yahoo.com
}

\begin{abstract}
Resumen. La fauna de murciélagos de Chiapas, una de las más estudiadas del país, representa cerca del 77\% de las especies registradas para México. Sin embargo, se ha prestado poca atención a los murciélagos de la región selva-norte, área convergente entre la Planicie del Golfo, la Sierra Chiapaneca y la porción más norteña de la Selva Lacandona. Con el objetivo de complementar el listado de las especies de la Región Selva Norte, entre febrero de 2008 y mayo de 2010 se capturaron murciélagos en 130 sitios mediante redes de niebla en Palenque, Chiapas. Los muestreos se realizaron en el continuo de vegetación del Parque Nacional Palenque (PNP) y en fragmentos aislados de vegetación en diferente grado de sucesión, así como en cercas vivas, corredores riparios y pastizales inducidos, localizados desde 1 hasta 14 kilómetros al norte del Parque. Con un esfuerzo de muestreo de $432 \mathrm{~m}$-red/noche, en 130 noches se registraron 52 especies de murciélagos; 10 catalogadas como especies hábitat-dependientes, 26 vulnerables a la fragmentación y 16 tolerables a la fragmentación. Entre las especies registradas se encuentran Noctilio leporinus, Mimon crenulatum, Chiroderma salvini, Myotis californicus, M. elegans, Rhogeessa tumida, Molosus rufus y M. sinaloae que no se habían registrado previamente en la zona. Los resultados de este trabajo muestran que la diversidad de murciélagos encontrada en Palenque representa el 42\% de la fauna de quirópteros mexicanos, por lo que el PNP y su periferia deben considerarse como área focal para la conservación de este grupo de mamíferos.
\end{abstract}

Palabras clave: riqueza de especies, fragmentación, corredores lineales, quiróptera.

\begin{abstract}
The bat fauna of Chiapas, is one of the most studied of Mexico, and representing about $77 \%$ of the bat species reported to the country. Although, low attention have received the bats from the Selva-Norte region (located in the Plains of the Gulf of Mexico, the Chiapas Mountains and the northern portion of Lacandona). With the objective of obtain a comprehensive list of the bat species of this region, we captured bats in 130 sites using mist-nets in Palenque Chiapas from February 2008 to May 2010. Capture sites were located in the large patch of natural vegetation that included the Palenque National Park (PNP), as well as isolated fragments of vegetation in different succession stages, live fences, riparian corridors and induced grasslands, located 1 to 14 kilometers north to the Park. With a sampling effort of 432 mist net hours/night, we recorded 52 bat species (10 species classified as habitat-dependent, 26 vulnerable to fragmentation and 16 fragmentation tolerant). Some of the not previously recorded in the Palenque area were Noctilio leporinus, Mimon crenulatum, Chiroderma salvini, Myotis californicus, M. elegans, Rhogeessa tumida, Molosus rufus and M. sinaloae. Results of this study show that the bat diversity found in Palenque, represents $42 \%$ of the Mexican bat fauna, making the PNP and its periphery, as an important area for the conservation of this mammalian group.
\end{abstract}

Key words: species richness, fragmentation, linear corridors, chiroptera.

\section{Introducción}

La quiropterofauna chiapaneca representa cerca del $77 \%$ de las especies reconocidas para México (Lorenzo y García, 2007; Medellín et al., 2008). Es también una de las mejor y más ampliamente estudiadas, pues existe un

Recibido: 24 enero 2011; aceptado: 23 junio 2011 buen número de trabajos sobre la diversidad del grupo, particularmente en la Región Selva-Centro (Medellín, 1993; Cruz et al., 2004; Escobedo-Morales et al., 2005), la Altiplanicie Central (Horváth et al., 2001; Naranjo y Medinilla, 2001), la Planicie Costera del Pacífico (Espinoza et al., 2003), la Sierra Madre de Chiapas (Espinoza et al., 1998) y además una revisión completa de los murciélagos del estado (Álvarez-Castañeda y Álvarez, 1991). 
No obstante, poco se conoce sobre la quiropterofauna de la Región Selva-Norte, área convergente entre la Planicie Costera del Golfo Sur, la Sierra Chiapaneca y la porción más norteña de la Selva Lacandona. Dentro de esta área, se incluye el Parque Nacional Palenque (PNP), donde se han registrado 42 especies de murciélagos (Horváth et al., 2006) que corresponden al 39.6\% de las 106 registradas para el estado (Lorenzo y García, 2007).

A pesar de la existencia del PNP, el continuo del bosque tropical perennifolio (BTP) que históricamente cubría el área (Rzendowsky, 2006), ha quedado reducido a un mosaico que supera los 300 fragmentos, compuesto de bosque tropical con distintos grados de aislamiento y perturbación (Díaz-Gallegos, 1996; Estrada et al., 2002). Aunado a esto, la zona de Palenque se encuentra rodeada de una gran diversidad de otros hábitats, tales como cercas vivas, corredores riparios y pastizales inducidos, que se complementan para producir un paisaje altamente heterogéneo.

La modificación y continua degradación de los bosques tropicales (Ortiz-Espejel y Toledo, 1998; Mendoza y Dirzo, 1999; Ochoa-Gaona et al., 2007) puede producir efectos negativos sobre las especies de murciélagos, como la reducción en número y calidad de los recursos que usan, incluyendo los sitios de refugio o alimentación (Kunz y Lumsden, 2003; Ortiz-Ramírez et al., 2006). Estos cambios pueden reflejarse en la disminución de su sobrevivencia y éxito reproductivo, y la exclusión de las comunidades de aquellas especies que dependen de hábitats mediana o altamente conservados (Galindo-González, 2004; Klingbeil y Willin, 2009; Schulze et al., 2000). Es así como la pérdida y fragmentación de hábitat modifica la composición y funcionalidad de las comunidades locales de murciélagos (Schulze et al., 2000; Quesada et al., 2004; Caras y Korine, 2009; Estrada-Villegas et al., 2010).

Es posible que debido a la plasticidad de las características biológicas y ecológicas de los murciélagos (Patterson et al., 2003), la heterogeneidad del paisaje en la zona de Palenque permita que especies de murciélagos tolerantes a los ambientes modificados coincidan con especies de hábitats mayormente conservados (Schulze et al., 2000; Galindo-González, 2004; Castro-Luna et al., 2007). Ante esta situación, en la comunidad de murciélagos de Palenque podría esperarse una combinación de especies con diversos grados de tolerancia a la fragmentación y pérdida de hábitat.

Los objetivos de este trabajo fueron 1), actualizar el listado de la quiróptero-fauna conocida para la zona de Palenque; 2), conocer la composición funcional del grupo según el gremio trófico y conducta de forrajeo y 3 ), clasificar las especies registradas de acuerdo con su respuesta a la degradación del hábitat.

\section{Materiales y métodos}

El área de estudio se localiza en el municipio de Palenque al noroeste del estado mexicano de Chiapas ( $\left.17^{\circ} 30^{\prime} 33^{\prime \prime} \mathrm{N}, 92^{\circ} 58^{\prime} 56^{\prime \prime} \mathrm{O}\right)$. Los sitios muestreados se distribuyeron en 2 zonas, el PNP y la porción noreste del municipio de Palenque hasta el ejido de San Mateo, considerada para fines de este trabajo como zona de influencia (de 1 a 14 kilómetros al noroeste del PNP; Fig. 1). Se excluyeron las regiones de la montaña al sur de Palenque, debido a conflictos sociales.

Fisiográficamente, la porción norte de Palenque pertenece a la Planicie Costera del Golfo Sur, mientras que en su porción centro-sur da inicio la Sierra Chiapaneca, con altitudes que van de 20 a $400 \mathrm{~m}$ snm. El clima es cálido-húmedo con temperatura promedio anual de $26^{\circ} \mathrm{C}$; alcanza $30^{\circ} \mathrm{C}$ en mayo y baja a $22^{\circ} \mathrm{C}$ durante diciembre. La precipitación media anual es de $2156 \mathrm{~mm}$ con lluvias concentradas particularmente de mayo a diciembre. Palenque tiene fuerte influencia de vientos norteños provenientes del golfo de México. La presencia de afluentes del río Usumacinta, como el Tulijá, Michol, Nututun y Chancalá forman una compleja red de ríos y arroyos en toda la zona (CEIGE, 2008).

En la zona centro y sur del área de estudio, la vegetación original dominante era bosque tropical perennifolio (BTP); en la zona norte dominaba el bosque tropical subperennifolio (BTS) y existe vegetación riparia (VR) asociada a los ríos y arroyos. Sin embargo, la apertura y extensión de terrenos agroganaderos ha causado la pérdida de poco más del 75\% de la vegetación original (Díaz-Gallegos, 1996; Estrada et al., 2002).

$\mathrm{El}$ área de estudio comprende diferentes elementos del paisaje, incluyendo parches de vegetación secundaria y áreas antropizadas. El PNP (Fig. 1) ocupa una superficie de 1771 ha, de las cuales únicamente 500 ha, corresponden al continuo de vegetación del BTP, caracterizado por especies arbóreas como Brosimum alicastrum, Poulsenia armata, Ficus mexicana, Manilkara zapota, Vatairea lundellii, Guatteria anomala y Quararibea funebris. En la zona se han registrado poco más de 400 especies entre árboles, arbustos, herbáceas, trepadoras, palmas y helechos (Días-Gallegos, 1996).

El resto de la zona comprende un gradiente de vegetación secundaria hasta los pastizales inducidos. En los parches con vegetación secundaria, de acuerdo con el tiempo de abandono, las especies más abundantes son de las familias Leguminosae, como Pithecellobium arboreum; Rubiaceae, como Hamelia patens; Moraceae, como Ficus mexicana; Cecropiaceae, como Cecropia obtusifolia; Tiliaceae, como Heliocarpus mexicanus; Solanaceae, como Solanum sp.; Euphorbiaceae, como Acalypha 


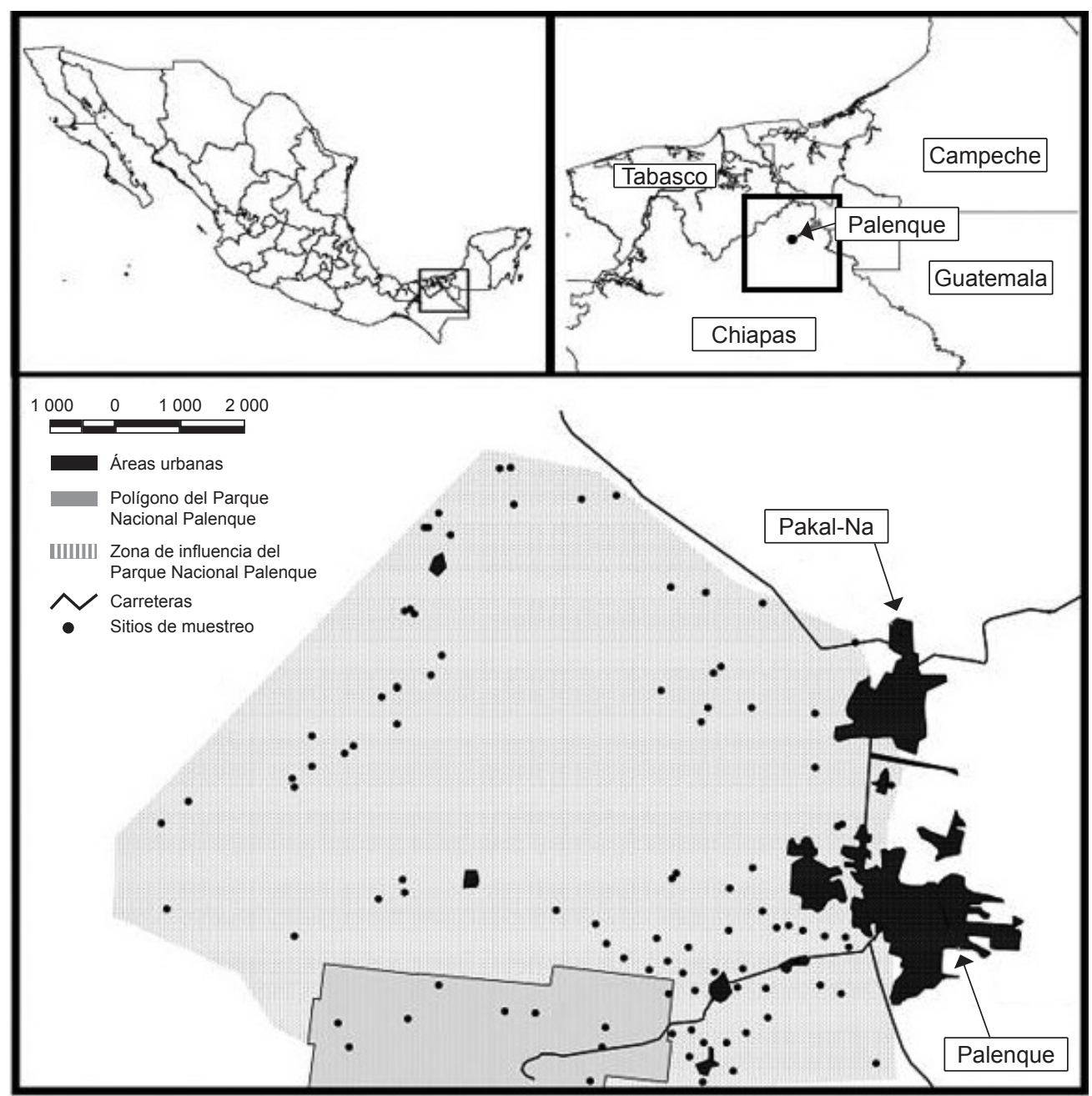

Figura 1. Localización de la zona de estudio en Palenque.

diversifolia, y Piperaceae, como Piper hispidum (DíazGallegos, 1996; Ochoa-Gaona et al., 2007).

Las áreas antropizadas caracterizan la zona de influencia del PNP. La vegetación original de esta zona ha sido desplazada principalmente por los pastizales inducidos, al dedicar la tierra a la ganadería extensiva. En estos pastizales se mantienen árboles aislados, como Ceiba pentandra, Ficus sp., y Pithecellobium sp. (Díaz-Gallegos, 1996; Jiménez-Ferrer et al., 2008a) y algunas especies forrajeras, como Guazuma ulmifolia y Gliricidia sepium (Jiménez-Ferrer et al., 2008b). Además de los pastizales inducidos, pueden encontrarse monocultivos de palma aceitera (Elaeis guineensis) y parches de encinos tropicales (Quercus oleoides).

Dentro de los pastizales inducidos se encuentran cercas vivas (CV), colocadas por los dueños de ranchos para delimitar zonas de pastoreo; son líneas de longitud y anchura variable con árboles de altura y follaje prácticamente similar, equidistantes uno de otro. Las componen especies arbóreas de rápido crecimiento como Bursera simaruba, Guazuma ulmifolia y Gliricidia sepium, también algunos árboles solitarios de talla mayor, como Ceiba pentandra $\mathrm{o}$ Ficus mexicana.

Los corredores riparios (CR) se conforman de vegetación asociada y localizada en la ribera de ríos y arroyos. Inmersos o anexos a los pastizales inducidos, los CR poseen forma y anchura en relación con el cauce de los arroyos y en algunos casos suelen verse seccionados para permitir el acceso del ganado al agua. En los CR se observan especies de árboles de 20 a 25 metros de altura o más, como Ficus mexicana, Pithecellobium arboreum, Lonchocarpus sp., Bursera simaruba y Spondia sp., asociadas con un numeroso grupo de arbustos, hierbas, epífitas y trepadoras, que en conjunto constituyen un componente estructuralmente complejo y potencialmente útil para la fauna asociada (de Lima y Gascon, 1999; Lees y Peres, 2007). 
Cuadro 1. Grupos funcionales en los que se asocian las especies de murciélagos de Palenque

\begin{tabular}{cccc}
\hline Hábitat de forrajeo & Modo forrajeo & Dieta & Grupo Funcional \\
\hline Espacios abiertos & Aéreo & Insectívoros & IAA \\
Entre vegetación & Aéreo & Insectívoros & IAV \\
Cerca a sustrato & Aéreo & Insectívoros & IAS \\
Sobre sustrato & Sustrato & Carnívoros & IS \\
Espacios cerrados & Sustrato & Piscívoros & C \\
Espacios abiertos/cerrado & Sustrato & Hematófagos & P \\
Espacios abierto/cerrado & Sustrato & Frugívoros & H \\
Entre dosel & Sustrato & Frugívoros & FD \\
Entre sotobosque & Sustrato & Frugívoros & FS \\
Dosel y sotobosque & General & Nectarívoros/polinívoros & FG \\
Espacios cerrados & Sustrato & Omnívoros & N/P \\
Espacios cerrados & Sustrato & O
\end{tabular}

Fuentes. Kalko, 1997; Schnitzer y Kalko; 1998, Sampaio et al., 2003 y Giannini y Kalko, 2004. Grupos funcionales. IAA, insectívoros aéreos que vuelan y cazan en espacios abiertos; IAV, insectívoros aéreos que vuelan y cazan entre la vegetación; IAS, insectívoros aéreos que vuelan y cazan cerca de sustrato; IS, insectívoros cazadores sobre el sustrato; C, carnívoros, P. piscívoros; H, hematófagos; $\mathrm{FD}$, frugívoros que vuelan en el dosel; FS, frugívoros que vuelan en el sotobosque; FG, frugívoros generalistas; N/P, nectarívoros polinívoros; O, omnívoros.

Selección de sitios y captura de murciélagos. En función del permiso de acceso a ranchos privados y terrenos ejidales, entre febrero de 2008 y mayo de 2010 se visitaron 130 sitios distribuidos a lo largo de los diferentes elementos del paisaje de Palenque, y separados por una distancia mínima de 500 $\mathrm{m}$ cada sitio. Se muestreó una sola noche durante 6 horas, activando las redes a partir de la puesta del sol. En cada sitio se utilizaron redes de niebla de diferente longitud (3, 6 y 12 $\mathrm{m})$, para tener un esfuerzo de muestreo estándar de $432 \mathrm{~m}$ de red por noche (6 redes de $12 \mathrm{~m}$ activas durante 6 horas). Las redes se colocaron a la orilla de la vegetación, cercanas al sustrato, dentro del sotobosque, cruzando arroyos o sobre cuerpos de agua, tratando siempre de cubrir las zonas de vuelo y forrajeo de los murciélagos. Las noches de muestreo fueron cerca o durante la luna nueva. La estacionalidad de la zona no fue un parámetro considerado para este trabajo.

Cada uno de los individuos capturados se colocó en un saco de manta, que se lavó después de cada uso. Los ejemplares se identificaron hasta nivel de especie, utilizando la clave de campo de Medellín et al. (2008). Para manipularlos se siguieron los lineamientos propuestos por Gannon et al. (2007). Una vez determinada la especie, los organismos fueron liberados. Para determinar el número potencial de especies de quirópteros en el área de Palenque en relación con el esfuerzo de muestreo se utilizó el estimador no paramétrico de Jacknife de segundo orden (Moreno, 2001).

Clasificación de las especies en grupos funcionales. La asignación de grupos funcionales se basó en los trabajos de Kalko (1997), Schnitzler y Kalko (1998), Sampaio et al. (2003) y Giannini y Kalko (2004). Las especies de murciélagos registradas se integraron en 12 grupos funcionales basados en 3 parámetros: 1), el hábitat de forrajeo, explicado por el espacio y medio acústico ocupado durante el vuelo;
2), el modo de forrajeo, que se refiere al área donde obtienen su alimento, ya sea sobre algún sustrato o en vuelo libre y 3), el tipo predominante de dieta, que es el tipo de alimento mayormente consumido por cada especie (Cuadro 1). Para esta sección, se integraron los listados de murciélagos de este trabajo y los obtenidos por Horváth et al. (2006) para la zona de Palenque.

Clasificación de murciélagos en relación con la fragmentación-perturbación del hábitat. Este análisis incluyó exclusivamente las especies registradas en el presente trabajo. Se excluyen las registradas por Horváth et al. (2006) porque las áreas de captura no están especificadas. Las especies se catalogaron según su respuesta a la fragmentación del bosque tropical, de acuerdo con Galindo-González (2004) y Estrada-Villegas et al. (2010). La clasificación incluye 3 categorías: $A$ ), especies restringidas o hábitat-dependientes; se encuentran únicamente en sitios con hábitats complejos en estructura y composición que pueden incluir cuerpos de agua $y / o$ vegetación secundaria en niveles avanzados de sucesión; $B$ ), especies sensibles o vulnerables; suelen mantenerse dentro del continuo de vegetación utilizando los $\mathrm{CR}$ y/o las CV para moverse entre fragmentos, sin alcanzar las zonas abiertas o sin vegetación y $C$ ), especies altamente tolerables o adaptables; son las generalistas, soportan la modificación del hábitat y suelen beneficiarse de tal transformación. Se pueden encontrar desde la selva madura hasta los pastizales inducidos.

\section{Resultados}

Quiropterofauna de Palenque. Se registraron 52 especies de murciélagos (Cuadro 2), con un esfuerzo total de muestreo de 56160 metros/red (en 130 noches) en un área 
cubierta en el muestreo de aproximadamente de $69.5 \mathrm{~km}^{2}$. El método de Jacknife de segundo orden, estima la riqueza en 57 especies de murciélagos.

Grupos funcionales. La fauna de murciélagos en Palenque se integra de 7 gremios funcionales, subdivididos en 12 categorías. Las especies insectívoras resultaron las más diversas con 30 especies (51.72\%), seguidas de 19 especies frugívoras $(32.75 \%), 4$ nectarívoras/polinívoras $(6.89 \%)$, y en menor proporción 2 especies del grupo de los hematófagos (3.44\%) y con una única representante por grupo, las omnívoras, carnívoras y piscívoras $(1.72 \%$ cada grupo). Este total incluye las especies registradas anteriormente en la zona por Horváth et al., 2006 (Cuadro 2).
Los gremios funcionales más diversos en Palenque los integran murciélagos insectívoros; se conforman por 16 especies IAS (por ej., Rhynchonycteris naso, Mormoops megalophylla, Myotis spp.), 6 especies son IAA ( por ej., Molossus molossus y M. sinaloae), 6 especies son IS (por ej., Lampronycteris brachyotis, Micronycteris microtis y Mimon crenulatum) y 2 especies son IAV (Natalus stramineus y Pteronotus parnellii). En el caso de los murciélagos frugívoros, 7 especies son FD ( por ej., Artibeus jamaicensis, A. lituratus y Chiroderma salvini), 8 especies FS ( por ej., Carollia spp. y Sturnira spp.) y 4 especies son FG por ej., Artibeus phaeotis y A. watsoni).

Clasificación de murciélagos en relación con la fragmentación-perturbación del hábitat. La clase $\mathrm{B}$, vulnerables

Cuadro 2. Listado taxonómico de los murciélagos de Palenque

\begin{tabular}{|c|c|c|c|c|c|c|}
\hline FAMILIA & ESPECIE & 2006 & 2010 & GREMIO & $R F r$ & $E P C$ \\
\hline \multirow[t]{6}{*}{ Emballoniridae } & Rhynchonycteris naso & $\mathrm{x}$ & $\mathrm{x}$ & IAS & $\mathrm{A}$ & $\mathrm{b}$ \\
\hline & Saccopteryx bilineata & $\mathrm{x}$ & $\mathrm{x}$ & IAS & A & \\
\hline & Balantiopteryx io & $\mathrm{x}$ & $\mathrm{x}$ & IAA & A & $\mathrm{a}$ \\
\hline & Balantiopteryx plicata & $\mathrm{x}$ & $\mathrm{x}$ & IAA & $\mathrm{B}$ & \\
\hline & Peropteryx macrotis & $\mathrm{x}$ & & IAS & - & \\
\hline & Peropteryx klappleri & $\mathrm{x}$ & & IAS & - & $\mathrm{b}$ \\
\hline Noctilionidae & Noctilio leporinus & & $\mathrm{x}$ & $\mathrm{P}$ & A & \\
\hline \multirow[t]{4}{*}{ Mormoopidae } & Mormoops megalophylla & $\mathrm{x}$ & $\mathrm{x}$ & IAS & $\mathrm{B}$ & \\
\hline & Pteronotus davyi & $\mathrm{x}$ & $\mathrm{x}$ & IAS & $\mathrm{B}$ & \\
\hline & Pteronotus parnellii & $\mathrm{x}$ & $\mathrm{x}$ & IAV & $\mathrm{B}$ & \\
\hline & Pteronotus personatus & $\mathrm{x}$ & $\mathrm{x}$ & IAS & $\mathrm{B}$ & \\
\hline \multirow[t]{25}{*}{ Phyllostomidae } & Glyphonycteris sylvestris & $\mathrm{x}$ & $\mathrm{x}$ & IS & A & a \\
\hline & Lampronycteris brachyotis & $\mathrm{x}$ & & IS & - & $\mathrm{b}$ \\
\hline & Micronycteris microtis & & $\mathrm{x}$ & IS & A & \\
\hline & Micronycteris schmidtorum & & $\mathrm{x}$ & IS & A & $\mathrm{b}$ \\
\hline & Desmodus rotundus & $\mathrm{x}$ & $\mathrm{x}$ & $\mathrm{H}$ & $\mathrm{C}$ & \\
\hline & Diphylla ecaudata & & $\mathrm{x}$ & $\mathrm{H}$ & $\mathrm{C}$ & \\
\hline & Chrotopterus auritus & $\mathrm{x}$ & & $\mathrm{C}$ & - & $\mathrm{b}$ \\
\hline & Mimon crenulatum & & $\mathrm{x}$ & IS & A & $\mathrm{b}$ \\
\hline & Phyllostomus discolor & $\mathrm{x}$ & $\mathrm{x}$ & $\mathrm{O}$ & $\mathrm{B}$ & \\
\hline & Lophostoma brasiliense & $\mathrm{x}$ & $\mathrm{x}$ & IS & $\mathrm{B}$ & $\mathrm{b}$ \\
\hline & Choeroniscus godmani & $\mathrm{x}$ & $\mathrm{x}$ & $\mathrm{N} / \mathrm{P}$ & $\mathrm{B}$ & $\mathrm{a}$ \\
\hline & Glossophaga commissarisi & & $\mathrm{x}$ & $\mathrm{N} / \mathrm{P}$ & $\mathrm{B}$ & \\
\hline & Glossophaga leachii & $\mathrm{x}$ & $\mathrm{x}$ & $\mathrm{N} / \mathrm{P}$ & $\mathrm{B}$ & \\
\hline & Glossophaga morenoi & $\mathrm{x}$ & $\mathrm{x}$ & $\mathrm{N} / \mathrm{P}$ & $\mathrm{C}$ & $a^{*}$ \\
\hline & Glossophaga soricina & $\mathrm{x}$ & $\mathrm{x}$ & $\mathrm{N} / \mathrm{P}$ & $\mathrm{C}$ & \\
\hline & Artibeus jamaicensis & $\mathrm{x}$ & $\mathrm{x}$ & FD & $\mathrm{C}$ & \\
\hline & Artibeus lituratus & $\mathrm{x}$ & $\mathrm{x}$ & FD & $\mathrm{C}$ & \\
\hline & Carollia perspicillata & $\mathrm{x}$ & $\mathrm{x}$ & FS & $\mathrm{C}$ & \\
\hline & Carollia subrufa & & $\mathrm{x}$ & FS & $\mathrm{B}$ & \\
\hline & Carollia sowelli & $\mathrm{x}$ & $\mathrm{x}$ & FS & $\mathrm{B}$ & \\
\hline & Centurio senex & $\mathrm{x}$ & $\mathrm{x}$ & FS & $\mathrm{B}$ & \\
\hline & Chiroderma salvini & & $\mathrm{x}$ & FD & $\mathrm{B}$ & \\
\hline & Chiroderma villosum & $\mathrm{x}$ & $\mathrm{x}$ & FD & $\mathrm{B}$ & \\
\hline & Artibeus azteca & $\mathrm{x}$ & & FG & - & \\
\hline & A. phaeotis & $\mathrm{x}$ & $\mathrm{x}$ & FG & B & \\
\hline
\end{tabular}


Cuadro 2. Continúa

\begin{tabular}{|c|c|c|c|c|c|c|}
\hline FAMILIA & ESPECIE & 2006 & 2010 & GREMIO & $R F r$ & $E P C$ \\
\hline & A. tolteca & & $\mathrm{x}$ & FG & $\mathrm{B}$ & \\
\hline & A. watsoni & $\mathrm{x}$ & $\mathrm{x}$ & FG & B & $\mathrm{b}$ \\
\hline & Platyrrhinus helleri & $\mathrm{x}$ & $\mathrm{x}$ & FG & $\mathrm{B}$ & \\
\hline & Sturnira lilium & $\mathrm{x}$ & $\mathrm{x}$ & FS & $\mathrm{C}$ & \\
\hline & Sturnira ludovici & $\mathrm{x}$ & $\mathrm{x}$ & FS & $\mathrm{C}$ & \\
\hline & Uroderma bilobatum & $\mathrm{x}$ & $\mathrm{x}$ & FS & $\mathrm{C}$ & \\
\hline & Vampyressa thyone & $\mathrm{x}$ & $\mathrm{x}$ & FD & $\mathrm{C}$ & \\
\hline & Vampyrodes caraccioli & $\mathrm{x}$ & $\mathrm{x}$ & FD & $\mathrm{C}$ & \\
\hline Natalidae & Natalus stramineus & & $\mathrm{x}$ & IAV & A & \\
\hline \multirow[t]{9}{*}{ Vespertilionidae } & Eptesicus furinalis & $\mathrm{x}$ & $\mathrm{x}$ & IAS & A & \\
\hline & Myotis californicus & & $\mathrm{x}$ & IAS & B & \\
\hline & Myotis elegans & & $\mathrm{x}$ & IAS & $\mathrm{B}$ & \\
\hline & Myotis fortidens & & $\mathrm{x}$ & IAS & B & a \\
\hline & Myotis keaysi & $\mathrm{x}$ & $\mathrm{x}$ & IAS & B & \\
\hline & Myotis nigricans & $\mathrm{x}$ & $\mathrm{x}$ & IAS & B & \\
\hline & Pipistrellus subflavus & $\mathrm{x}$ & & IAS & - & \\
\hline & Rhogeessa parvula & & $\mathrm{x}$ & IAS & B & $a^{*}$ \\
\hline & Rhogeessa tumida & & $\mathrm{x}$ & IAS & $\mathrm{B}$ & \\
\hline \multirow[t]{4}{*}{ Molossidae } & Cynomops mexicanus & $\mathrm{x}$ & $\mathrm{x}$ & IAA & $\mathrm{C}$ & $\mathrm{b}$ \\
\hline & Molossus molossus & $\mathrm{x}$ & $\mathrm{x}$ & IAA & $\mathrm{C}$ & \\
\hline & Molossus rufus & $\mathrm{x}$ & $\mathrm{x}$ & IAA & $\mathrm{C}$ & \\
\hline & Molossus sinaloae & & $\mathrm{x}$ & IAA & $\mathrm{C}$ & \\
\hline
\end{tabular}

Columna 2006, especies registradas en Horváth et al.(2006); columna 2010, registradas en este trabajo. Gremio(grupos funcionales). IAA, insectívoros aéreos que vuelan y cazan en espacios abiertos; IAV, insectívoros aéreos que vuelan y cazan entre la vegetación; IAS, insectívoros aéreos que vuelan y cazan cerca de sustrato; IS, insectívoros cazadores sobre el sustrato; C, carnívoros, P. piscívoros; $\mathrm{H}$, hematófagos; FD, frugívoros que vuelan en el dosel; FS, frugívoros que vuelan en el sotobosque; FG, frugívoros generalistas; N/P, nectarívoros polinívoros; $\mathrm{O}$, omnívoros. $R F r$ ( respuesta de la especie a la fragmentación del hábitat). A, hábitat-dependiente; B, vulnerable; C, adaptable ( tomado de Galindo-González, 2004 y Estrada-Villegas et al., 2010); EPC (especie con prioridad de conservación). a, en UICN; b, en NOM-059-SEMARNAT-2010; * endémicas de México.

a la fragmentación, domina la comunidad de murciélagos de Palenque con 26 especies, como Pteronotus personatus, Phyllostomus discolor y Myotis keaysi. La clase A, hábitatdependientes, se integra por 10 especies de murciélagos, como Saccopteryx bilineata, Rhynchonycteris naso y Mimon crenulatum. La clase C, murciélagos tolerables a la fragmentación, se compone de 16 especies, entre otras, Glossophaga soricina, Artibeus lituratus y Sturnira lilium.

\section{Discusión}

En la zona de Palenque se habían registrado 42 especies de murciélagos, mediante el uso de redes de niebla y búsqueda e inspección de refugios (Horváth et al., 2006). De esas 42 especies anteriormente registradas, 6 no lograron capturarse durante este estudio. Al combinar las especies de la lista de Horváth et al. (2006) con las registradas en este trabajo, la fauna de murciélagos de Palenque estaría compuesta por 58 especies de murciélagos, agrupadas en 34 géneros y 7 familias. De estas especies, 16 son nuevos registros para Palenque (Cuadro 2).
Los murciélagos de Palenque constituyen el $42 \%$ de la quiropterofauna reconocida para México (Medellín et al., 2008), y el $54.7 \%$ de los registrados en el estado de Chiapas (Lorenzo y García, 2007). Están consideradas en algún nivel de riesgo, amenaza o bajo protección 15 especies registradas en la zona, que incluyen Rhogeessa parvula y Glossophaga morenoi (especies endémicas de México; Hutson et al., 2001; SEMARNAT, 2010).

Dada la similitud con otros sitios, es posible que al cubrir la zona sur del PNP, mediante el uso de redes de niebla, pudieran adicionarse a la lista especies como, Trachops cirrhosus, Mimon cozumelae e Hylonycteris underwoodi. $\mathrm{Si}$ el uso de las redes de niebla se complementara con la búsqueda e inspección en refugios, probablemente también podrían registrarse en la zona especies como Pteronotus gymnonotus y Natalus lanatus, de cuyas familias (Mormoopidae y Natalidae, respectivamente) se sabe que tienen preferencias cavernícolas o son mayormente encontradas en cavidades (Escalona-Segura et al., 2002; Avila-Torresagatón, 2008; Torres-Flores y López-Wilchis, 2010). Además, el uso de los métodos de detección acús- 
tica podría complementar los listados, como ha ocurrido en otras regiones neotropicales (O'Farrell y Miller, 1999; Ochoa et al., 2000; Rydell et al., 2002).

En la zona de Palenque, mediante el uso de detectores acústicos y de los mapas de distribución trazados por Medellín et al. (2008) sería posible registrar especies como Centronycteris centralis, Pteronotus gymnonotus, Lasiurus blossevillii, L. ega, L. intermedius, Rhogeessa gracilis, Eumops auripendulus, E. bonariensis, E. glaucinus, E. hanse, Promops centralis y Nyctinomops laticaudatus.

En la fauna de murciélagos de Palenque domina la familia Phyllostomidae (56.8\% de las especies registradas), siguiendo el patrón de otras comunidades de murciélagos neotropicales (Fleming, 1986; Stevens y Willig, 2000; Kalko y Handley Jr., 2001). El gremio trófico dominante es el insectívoro, con cerca del $52 \%$ de las especies registradas, seguido por los frugívoros (el 32.7\% de las especies). Esto mismo se ha observado en Belice (Fenton et al., 2001), Brasil y Costa Rica (Stevens y Willig, 2000; Sampaio et al., 2003). Sin embargo, en algunas localidades de Panamá (Meyer et al., 2008) y Brasil (Kalko y Handley Jr., 2001), el gremio de murciélagos frugívoros es el dominante. En las regiones neotropicales se han registrado ambos patrones (Patterson et al., 2003).

Jung y Kalko (2010) anotan que los murciélagos insectívoros aéreos pueden soportar ambientes con alteraciones antrópicas. Sin embargo, la presencia de diferentes especies de murciélagos insectívoros en sitios con distintos niveles de perturbación humana podría estar modulada tanto por la condición del hábitat como por la disponibilidad de insectos atraídos por la modificación gradual de los sistemas originales (Basset et al., 2004; Jung y Kalko, 2010). En este sentido, la alta diversidad de murciélagos insectívoros de Palenque se podría explicar por la también alta diversidad de insectos del BTP de Chiapas (Cordero y Llorente, 2000; León-Cortés et al., 2005).

Los diferentes elementos del paisaje de Palenque, resultantes de la fragmentación y pérdida del BTP, parecen modular la presencia de las diferentes especies de murciélagos en los componentes paisajísticos de la zona, es decir, la respuesta de este grupo biológico parece depender de las características de los diferentes elementos del paisaje, como su cobertura, su estructura, la continuidad de BTP o el grado de conectividad entre fragmentos (Galindo-González, 2004; Estrada-Villegas et al., 2010; Mena, 2010). Es probable que lo anterior se encuentre estrechamente vinculado a las historias de vida de cada especie de murciélago, en particular de sus requerimientos energéticos (alimento) y disponibilidad de refugio (Kunz y Lumsden, 2003; Patterson et al., 2003; Speakman y Thomas, 2003).

A partir del listado de Galindo-González (2004), Sturnira ludovici y Vampyressa thyone, consideradas especies clase A, se reasignan a la clase C; Glossophaga morenoi, clasificada como clase B, a la clase C, y Mormoops megalophylla, Pteronotus davyi, Chiroderma salvini y C.villosum, pertenecientes a la clase $\mathrm{C}$, se reasignaron a la clase $B$, toda vez que fueron registradas en ambientes que no reflejan las características paisajísticas de la clasificación original (Galindo-González, 2004). Es decir, algunas especies de murciélagos, típicas de hábitats conservados, como el bosque continuo o los $\mathrm{CR}$, fueron capturadas en elementos del paisaje con alta influencia humana, como son las CV o los PI.

En Palenque, la modificación del hábitat por acciones humanas pudiera favorecer a las especies clase C (la mayoría de ellas registradas en todos los elementos del paisaje, particularmente en las zonas altamente perturbadas como los PI). Mientras que las especies de murciélagos cuya respuesta a la fragmentación las sitúa como hábitat-dependientes (clase A y B) serían en un principio las mayormente afectadas por la fragmentación y modificación del BTP de la zona.

Basta mencionar que 7 de las 15 especies de murciélagos encontradas en Palenque y catalogadas en algún nivel de riesgo (Hutson et al., 2001; SEMARNAT, 2010) pertenecen a la clase A (por ej., Balantiopteryx io, Rhynchonycteris naso, Glyphonycteris sylvestris o Micronycteris schmidtorum; Cuadro 2) y 3 a la clase B (Choeroniscus godmani, Myotis fortidens o la endémica Rhogeessa párvula). En contraste, sólo 2 especies pertenecen a la clase C (Cynomops mexicanus y Glossophaga morenoi; endémica esta última).

Los CR y las CV son hábitats importantes para el mantenimiento de las poblaciones de murciélagos en paisajes fragmentados (Verboom y Huitena, 1997; Estrada y Coates-Estrada, 2001; Harvey et al., 2006; Rogers et al., 2006; Williams et al., 2006; Harvey y González, 2007). En estos hábitats, los murciélagos desarrollan diversas fases de su ciclo de vida y tienen acceso a sitios de percha, refugios diurnos y alimento (Kunz y Lumsden, 2003; Dechmann y Kerth, 2008). Por esta razón, resulta necesario diseñar y poner en marha estrategias de mejoramiento de la composición y estructura de las cercas vivas entre pastizales inducidos y del mantenimiento de los corredores riparios dentro del paisaje de Palenque.

Debido a la acelerada y continua fragmentación y degradación del BTP que ocurre en Palenque, es posible que parte importante de su patrimonio biológico disminuya drástica e irreversiblemente en un futuro cercano. Arita y Ortega (1998) consideran la región de la Selva Lacandona como importante y prioritaria para la conservación de la quiropterofauna mesoamericana. Asimismo, Medellín (1993) considera la región este de Tabasco y la Selva Lacandona como zona relevante e importante para 
la permanencia de varias especies de este grupo. En este sentido, la zona de Palenque además de formar parte del continuo de vegetación de la Selva Lacandona (Patten et al., 2010) y colindar con el este de Tabasco, debe considerarse zona prioritaria para el estudio y conservación de los murciélagos de México, pues alberga poco más del $40 \%$ de esta fauna.

\section{Agradecimientos}

La captura de ejemplares se hizo bajo el permiso SGPA/DGVS/05491/09 SEMARNAT. Se agradece a la Comisión Nacional de Áreas Naturales Protegidas, por el financiamiento del proyecto "Estudio de la viabilidad para el establecimiento de corredores biológicos para la conservación de la fauna silvestre en la zona de influencia de Palenque". Al INAH-Palenque y CONANP-Palenque, por la facilidades para trabajar en el PNP. A los dueños de los ranchos por permitirnos el acceso, así como a Acajungla y el parque Los Aluxes. La División Académica de Ciencias Biológicas-Universidad Juárez Autónoma de Tabasco (DACBiol-UJAT) proporcionó apoyo logístico para el desarrollo de este trabajo. A los estudiantes de la DACBiol $\mathrm{y}$ voluntarios que apoyaron el trabajo de campo. A tres revisores anónimos que mejoraron el documento final. El primer autor recibió la beca 31864 del CONACyT para cursar la maestría en ciencias ambientales (DACBiol-UJAT).

\section{Literatura citada}

Álvarez-Castañeda, S. T. y T. Álvarez. 1991. Los murciélagos de Chiapas. Instituto Politécnico Nacional. México D. F. 211 p.

Arita, T. H. y J. Ortega. 1998. The middle american bat fauna: conservation in the neotropical-nearctic border. In Bats biology and conservation, T. H. Kunz y P. A. (eds.). Smithsonian Institution Press, Washington, D.C. p. 295-308.

Avila-Torresagatón, L. G. 2008. Estimación mediante un sistema audiovisual de las poblaciones de 4 especies de murciélagos en 5 refugios cavernícolas de la Reserva de la Biosfera Sierra de Huautla, Estado de Morelos, México. Tesis, Facultad de Ciencias Biológicas, Universidad Autónoma del Estado de Morelos. Cuernavaca, Morelos. 78 p.

Basset, Y., J. F. Mavoungou, J. B. Mikissa, O. Missa, S. Miller, R. Kitching y A. Alonso. 2004. Discriminatory power of different arthropod data sets for the biological monitoring of anthropogenic disturbance in tropical forest. Biodiversity and Conservation 13:709-732.

Caras, T. y C. Korine. 2009. Effect of vegetation density on the use of trails by bats in a secondary tropical rain forest. Journal of Tropical Ecology 25:97-101.

Castro-Luna, A., V. J. Sosa y G. Castillo-Campos. 2007. Quantifying phyllostomid bats at different taxonomic levels as ecological indicators in a disturbed tropical forest. Acta Chiropterologica 9:219-228.

Comité Estatal de Información Geográfica y Estadística (CEIGE). 2008. http://www.ceieg.chiapas.gob.mx; última consulta: 11.II. 2010

Cordero, C. M. y J. Llorente. 2000. Los Arthropoda de México: algunas comparaciones. In Biodiversidad, taxonomía y biogeografía de artrópodos de México: hacia una síntesis de su conocimiento, vol. II, J. E. Llorente-Bousquets, A. N. García-Aldrete, E. González-Soriano (eds.).UNAM. México. p. 95-101.

Cruz, E. L., C. Lorenzo, L. Soto, E. Naranjo y N. Ramírez. 2004. Diversidad de mamíferos en cafetales y selvas medianas de las cañadas de La Selva Lacandona, Chiapas, México. Acta Zoológica Mexicana 20:63-81.

de Lima, G. y C. Gascon. 1999. The conservation value of linear forest remnants in central Amazonia. Biological Conservation 91:241-247.

Dechmann, K. N. D. y G. Kerth. 2008 My home is your castle: roost making is sexually selected in the bat Lophostoma silvicolum. Journal of Mammalogy 89:1379-1390.

Díaz-Gallegos, J. R. 1996. Estructura y vegetación florística del Parque Nacional Zona Arqueológica de Palenque, Chiapas. Tesis, División Académica de Ciencias Biológicas. Universidad Juárez Autónoma de Tabasco,Villahermosa. 63 p.

Escalona-Segura, G. J., A. Vargas-Contreras y L. Interián-Sosa. 2002. Registros importantes de mamíferos para Campeche, México. Revista Mexicana de Mastozoología 6:99-103.

Escobedo-Morales, L. A., L. León-Paniagua, J. Arroyo-Cabrales y J. O. Polanco. 2005. Diversidad y abundancia de los mamíferos de Yaxchilán, municipio de Ocosingo, Chiapas. In Contribuciones mastozoológicas en homenaje a Bernardo Villa, V. Sánchez-Cordero y A. R. Medellín (eds.). Instituto de Biología, UNAM/ CONABIO. México DF. p. 283-298.

Espinoza, E., E. Cruz, H. Kramsky e I. Sánchez. 2003. Mastofauna de la Reserva de la Biosfera "La Encrucijada", Chiapas. Revista Mexicana de Mastozoología 7:5-19.

Espinoza, M. E., A. D. Anzures y E. A. Cruz. 1998. Mamíferos de la Reserva de la Biosfera El Triunfo, Chiapas. Revista Mexicana de Mastozoología 3:79-94.

Estrada, A. y R. Coates-Estrada. 2001. Bat species richness in live fences and in corridors of residual rain forest vegetation at Los Tuxtlas, Mexico. Ecography 24:92-102.

Estrada, A., A. Mendoza, L. Castellanos, R. Pacheco, S. Van Belle, Y. García y D. Muñoz. 2002. Population of the black howler monkey (Aluatta pigra) in a fragmented landscape in Palenque, Chiapas, México. American Journal of Primatology 58:45-55.

Estrada-Villegas, S., C. F. J. Meyer y E. Kalko. 2010. Effects of tropical forest fragmentation on aerial insectivorous bats in land-bridge island system. Biological Conservation 
143:597-608.

Fenton, M. B., E. Bernard, S. Bouchard, L. Hollis, S. D. Johnston, C. L. Lausen, J. M. Rataliffe, D. K. Riskn, J. R. Taylor y J. Zigouris. 2001. The bat fauna of Lamanai Belize: roost and trophic roles. Journal of Tropical Ecology 17:511-524.

Fleming, H. T. 1986. The structure of neotropical bat communities: a preliminary analysis. Revista Chilena de Historia Natural 59:135-150.

Galindo-González, J. 2004. Clasificación de los murciélagos de la región de Los Tuxtlas, Veracruz, respecto a su respuesta a la fragmentación del hábitat. Acta Zoológica Mexicana 20:293-243.

Gannon, W. L., S. R. Silkes y The animal care and use committee of the American Society of Mammalogists. 2007. Guidelines of the American Society of Mammalogists for the use of wild animals in research. Journal of Mammalogy 88:809-823.

Giannini, P. N. y E. Kalko. 2004. Trophic structure in a large assemblage of phyllostomid bats in Panama. Oikos 105:209220.

Harvey, A. C., A. Medina, D. Merlo, S. Vílchez, B. Hernández, J. Saenz, J. Maes, F. Casanoves y F. Sinclair. 2006. Patterns of animal diversity in different forms of tree cover in agricultural landscapes. Ecological Applications 16:1986-1999.

Harvey, A. C. y J. A. González. 2007. Agroforestry systems conserve species-rich but modified assemblages of tropical birds and bats. Biodiversity and Conservation 16:2257-2292.

Horváth, A., R. Vidal-López y R. Sarmiento-Aguilar. 2001. Mamíferos del Parque Nacional Lagunas de Montebello, Chiapas, México. Revista Mexicana de Mastozoología 5:626.

Horváth, A., R. Vidal-López, O. Pérez-Macias, C. Chávez-Gloria, Y. Aguirre-Bonifaz, D. Gallegos-Castillo, M. RamírezLozano, E. Sánchez-Vásquez y E. Espinoza-Medinilla. 2006. Mamíferos de los Parques Nacionales Lagunas de Montebello y Palenque Chiapas, informe final del proyecto BK047 CONABIO-ECOSUR. San Cristóbal de las Casas, Chiapas. 46 p.

Hutson, A., S. P. Mickleburgh y P. A. Racey. 2001. Global status and survey conservation action plan: microchiropteran Bats. IUCN/ SSC Chiropteran Specialist Group, Gland/ Cambridge. 258 p.

Jiménez-Ferrer, G., C. L. López, T. J. Nahed, G. S. Ochoa, G. S. y B. de Jong. 2008a. Árboles y arbustos forrajeros de la región norte-tzotzil de Chiapas, México. Veterinaria México 39:199-213.

Jiménez-Ferrer, G., R. Velasco-Pérez, G. M. Uribe y L. SotoPinto. 2008b. Ganadería y conocimiento local de árboles y arbustos forrajeros de la selva Lacandona, Chiapas, México. Zootecnia Tropical 26:333-337.

Jung, K. y E. Kalko. 2010. Where forest meets urbanization: foraging plasticity of aerial insectivorous bats in an anthropogenically altered environment. Journal of
Mammalogy 91:144-153.

Kalko, E. 1997. Diversity in tropical bats. In Tropical biodiversity and Systematics, H. Ulrich (ed.). Zoologisches Forschungsinstitut und Museum Alexander Koening Bonn. p. 13-43.

Kalko, E. y C. O Handley Jr. 2001. Neotropical bats in the canopy: diversity, community structure, and implication for conservation. Plant Ecology 153:319-333.

Klingbeil, T. B. y M. R. Willg. 2009. Guild-specific response of bats to landscape composition and configuration in fragmented Amazonian rainforest. Journal of Applied Ecology 46:203-213.

Kunz, H. T. y L. F. Lumsden. 2003. Ecology of cavity and foliage roosting bats. In Ecology of bats, T. H. Kunz y M. B. Fenton (eds.). University of Chicago Press. Illinois. p. 3-89.

Lees, A. C. y A. C. Peres. 2007. Corridors of varying quality for Amazonian birds and mammals. Conservation Biology 22:439-449.

León-Cortez, J. L, L. Ruiz-Montoya y A. Morón-Ríos. 2005. La diversidad de insectos en Chiapas: génesis y estado del conocimiento. In Diversidad biológica en Chiapas, M. Gonzales-Espinoza, N. Ramírez-Marcial, L. Ruiz-Montoya (coord.). ECOSUR-COCyTECH, Plaza y Valdés. México, D. F. p. 163-194.

Lorenzo, C. y M. García. 2007. Sistemática y conservación de los mamíferos de Chiapas. In Tópicos en sistemática, ecología y conservación de mamíferos, G. Sánchez-Rojas y A. Rojas-Martínez (eds.). Universidad Autónoma del Estado de Hidalgo, Pachuca, Hidalgo. p. 47-57.

Medellín, R. A. 1993. Estructura y diversidad de una comunidad de murciélagos en el trópico húmedo mexicano. In Avances en el estudio de los mamíferos de México, vol. II, Publicaciones Especiales. R. A. Medellín y G. Ceballos (eds.). Asociación Mexicana de Mastozoología, México, D. F. p. 333-350.

Medellín, R. A., H. Arita y O. Sánchez. 2008. Identificación de los murciélagos de México, clave de campo, segunda edición. Instituto de Ecología, UNAM, México, D. F. 78 p.

Mena, J. L. 2010. Respuesta de los murciélagos a la fragmentación del bosque en Pozuzo, Perú. Revista Peruana de Biología 17:277-284.

Mendoza, E. y R. Dirzo. 1999. Deforestation in Lacandonia (southeast Mexico): evidence for the declaration of the northernmost tropical hot-spot. Biodiversity and Conservation 8:1621-1641.

Meyer, C. F. J., J. Fründ, W. Pineda y E. Kalko. 2008. Ecological correlates of vulnerability to fragmentation in neotropical bats. Journal of Applied Ecology 45:381-391.

Moreno, C. E. 2001. Métodos para medir la biodiversidad, vol. 1. M\&T- Manuales y Tesis. SEA, Zaragoza. 84 p.

Naranjo, E. J. y E. E. Medinilla. 2001. Los mamíferos de la Reserva Ecológica Huitepec, Chiapas, México. Revista Mexicana de Mastozoología 5:58:67. 
O'Farrell, M. J. y B. M. Miller. 1999. Use of vocal signatures for the inventory of free-flying neotropical bats. Biotropica 31:507-516.

Ochoa, J., M. J. O'Farrell y B. M. Miller. 2000. Contribution of acoustic methods to the study of insectivorous bat diversity in protected areas from northern Venezuela. Acta Chiropterologica 2:171-183.

Ochoa-Gaona, S., F. Hernández-Vázquez, B. H. J. De Jong y D. Gurri-García. 2007. Pérdida de diversidad florística ante un gradiente de intensificación del sistema agrícola de rozatumba-quema: un estudio de caso en La Selva Lacandona, Chiapas, México. Boletín de la Sociedad Botánica de México 81:65-80.

Ortiz-Espejel, B. y V. M. Toledo. 1998. Tendencias en la deforestación de la Selva Lacandona: (Chiapas, México), el caso de las Cañadas. Interciencia 23:318-327.

Ortiz-Ramírez, D., C. Lorenzo, E. Naranjo y L. LeónPaniagua. 2006. Selección de refugios por 3 especies de murciélagos frugívoros (Chiroptera: Phyllostomidae) en la Selva Lacandona, Chiapas, México. Revista Mexicana de Biodiversidad 77:261-270.

Patten, A. M., H. Gómez y B. Smith-Patten. 2010. Long-term changes in the bird community of Palenque, Chiapas, in response to rainforest loss. Biodiversity and Conservation 19:21-36.

Patterson, B. D., M. R. Willig y D. Stevens. 2003. Trophic strategies, niche partitioning and patterns of ecological organization. In Ecology of bats, T. H. Kunz y M. B. Fenton (eds.). University of Chicago Press. p. 536-579.

Quesada, M., K. E. Stoner, J. A. Lobo, Y. Herrarías-Diego, C. Palacios-Guevara, M. A. Munguía-Rosas, K. O-Salazar y V. Rosas-Guerrero. 2004. Effects of forest fragmentation on pollinator activity and consequences for plant reproductive success and mating patterns in bat-pollinated Bombacaceous trees. Biotropica 36:131-138.

Rogers, D., M. C. Belk, M. González y B. Coleman. 2006. Pattersns of habitat use by bats along riparian corridor in northern Utah. The Southwestern Naturalist 51:51-58.

Rydell, J., H. Arita, M. Santos y J. Granados. 2002. Acoustic identification of insectivorous bats (Chiroptera) of Yucatán, Mexico. Journal of Zoology 257:27-36.
Rzedowsky J. 2006 Vegetación de México, edición digital, Comisión Nacional para el Conocimiento y Uso de la Biodiversidad, México, D. F. 504. p.

Sampaio, E. M., E. Kalko, E. Bernard, B. Rodríguez-Herrera y O. Handley Jr. 2003. A biodiversity assessment of bats (Chiroptera) in a tropical lowland rainforest of Central Amazonia, including methodological and conservation considerations. Studies on Neotropical Fauna and Environment 38:17-31.

Schnitzler, H. U. y E. Kalko. 1998. How echolocating bats search and find food. In Bats biology and conservation, T. H. Kunz y P. A. Racey (eds.). Smithsonian Institution Press, Washington, D.C. p. 183-196.

Schulze, D. M., N. E. Saevy y D. Whitecre. 2000. A comparison of the phyllostomid bat assemblages in undisturbed neotropical forest and in forest fragments of a slash-and-burn farming mosaic in Petén, Guatemala. Biotropica 32:174-184.

SEMARNAT (Secretaría de Medio Ambiente y Recursos Naturales). 2010. Norma Oficial Mexicana NOM-059SEMARNAT-2010. Protección ambiental, Especies nativas de México de flora y fauna silvestre, Categorías de riesgo y especificaciones para su inclusión, exclusión o cambio, Lista de especies en riesgo. Diario Oficial de la Federación, 30 de diciembre. $78 \mathrm{p}$.

Speakman J. R. y D. W. Thomas. 2003. Physiological ecology and energetics of bats. In Bats Biology and Conservation, T. H. Kunz y P. A. Racey (eds.). Smithsonian Institution Press, Washington, D.C. p. 430-490.

Steven, R. D. y M. R. Willig. 2000 Density compensation in New World bat communities. Oikos 98:367-377.

Torres-Flores J. y R. López-Wilchis. 2010. Condiciones microclimáticas, hábitos de percha y especies asociadas a los refugios de Natalus stramineus en México. Acta Zoológica Mexicana 26:191-213.

Verboom, B. y H. Huitema. 1997. The importance of linear landscape elements for the pipistrelle Pipistrellus pipistrellus and serotine bat Eptesicus serotinus. Landscape Ecology 12:117-125.

Williams J.A., M. O'Farrell y B. R. Riddle. 2006. Habitat use by bats in a riparian corridor of the Mojave Desert southern Nevada. Journal of Mammalogy 87:1145-1153. 